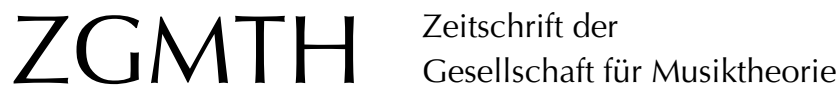

Bitzan, Wendelin (2012): Sur le mode mineur - Ein Mondbild in Moll. Eine analytische Annäherung an Claude Debussys Verlaine-Lied Clair de lune. ZGMTH 9/2, 289-301. https://doi.org/10.31751/684

(C) 2012 Wendelin Bitzan

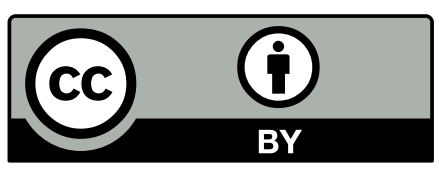

Dieser Text erscheint im Open Access und ist lizenziert unter einer Creative Commons Namensnennung 4.0 International Lizenz.

This is an open access article licensed under a Creative Commons Attribution 4.0 International License.

veröffentlicht / first published: 15/03/2013

zuletzt geändert / last updated: 04/09/2014 


\title{
Sur le mode mineur - Ein Mondbild in Moll Eine analytische Annäherung an Claude Debussys Verlaine-Lied Clair de lune
}

\author{
Wendelin Bitzan
}

\begin{abstract}
Eine Untersuchung der Tonsprache Debussys erfordert die Beachtung der ästhetischen und interdisziplinären Rahmenbedingungen impressionistischen Komponierens. In besonderem Maße gilt dies für die zahlreichen Werke, denen die Lyrik Paul Verlaines - als vertonter Text oder als Inspirationsquelle - zu Grunde liegt. Die Analyse der zweiten Fassung des Liedes Clair de lune (1891) erweist sich als aufschlussreich im Hinblick auf die Parameter der Melodik, Harmonik, Form und Syntax und erlaubt Rückschlüsse auf den Umgang Debussys mit Textvorlagen des literarischen Symbolismus.
\end{abstract}

Wer in musikalischen Werken des Fin de siècle nach Querverbindungen zum Schaffen von Zeitgenossen oder nach Verweisen auf andere Künste sucht, wird schnell fündig. Gerade der Impressionismus ist nach allgemeinem Verständnis undenkbar ohne außermusikalische Inspiration und programmatische Beziehungen. Der poetische Topos der Nacht und Dunkelheit, wie er im musikalischen Nachtstück Nocturne oder Notturno seinen Ausdruck findet, ist verschiedentlich als zentral für die romantische Ästhetik beschrieben worden. ${ }^{1}$ Noch bei Claude Debussy finden sich Anknüpfungspunkte an diese Tradition. Eine Reihe von Werken ist durch die Thematisierung des Mondlichts miteinander verbunden - dabei handelt es sich bevorzugt um Kompositionen, die in Zusammenhang mit der symbolistischen Dichtung Paul Verlaines stehen, dem von Debussy am häufigsten vertonten Dichter. Dieser Aufsatz behandelt exemplarisch Debussys Vertonung des Gedichts Clair de lune (1891) aus unterschiedlichen analytischen Perspektiven.

\section{Entstehungsgeschichtlicher Hintergrund}

Der junge Verlaine veröffentlichte im Jahr 1869 den Gedichtzyklus Fêtes galantes, eine Serie von einundzwanzig Gedichten, inspiriert durch den Rokokomaler Antoine Watteau. ${ }^{2}$ Der Terminus sfête galante ‘ als Sinnbild für eine aristokratische Festszene wird von Watteau als Gattungsbegriff verwendet und bezeichnet Gemälde mit einem spezifischen motivischen Ambiente, darunter höfische Feierlichkeiten, Schäferstücke oder Szenenbil-

1 Seidel 1969, 635 f. sowie Schmidt 1991, $83 \mathrm{ff}$.

2 Zum vielschichtigen Bedeutungsgehalt von Verlaines Zyklus siehe Hardeck 1967, 52 f. 
der der Commedia dell'arte. ${ }^{3}$ Als literarischer Zyklus gelangten Verlaines Fêtes galantes zu einiger Bekanntheit, und neben einigen anderen Komponisten nahm sich vor allem Claude Debussy der Gedichte an. Clair de lune, das sanft-melancholische erste Gedicht des Zyklus', spielt dabei eine besondere Rolle: Debussy vertonte es erstmals im Jahr 1882 gemeinsam mit einigen anderen Versen aus den Fêtes galantes und setzte es später ein zweites Mal, um es innerhalb einer dreiteiligen Liederserie unter gleichem Titel zu veröffentlichen. ${ }^{4}$ Die hier besprochene spätere Fassung des Liedes steht in den Fêtes galantes, $1^{\text {er }}$ recueil von 1891 an dritter Stelle und folgt auf En sourdine und Fantoches, die Gedichte Nr. 21 und Nr. 11 aus Verlaines Sammlung.

Die imaginativen Topoi des Mondes und Mondscheins tauchen einige Male in Instrumentalwerken Debussys auf; ebenso verhält es sich mit Verweisen auf die Ideenwelt und Atmosphäre der Fêtes galantes. Dass sich der Titel des Klavierstücks Clair de lune, des dritten Satzes der Suite bergamasque (1890), ebenfalls auf Verlaines Gedicht bezieht, gilt als gesichert. ${ }^{5}$ Umstritten ist hingegen, ob es die lautmalerischen, kaum übersetzbaren »masques et bergamasques" aus der zweiten Gedichtzeile von Clair de lune waren, die den Komponisten zur Betitelung der Suite inspirierten, oder ob die Namensgebung lediglich auf das Umland des norditalienischen Bergamo, einer einstigen Hochburg der Commedia dell'arte, verweist. ${ }^{6}$ Eine auf den Fêtes galantes basierende Ballettmusik, deren Komposition Debussy um 1910 beabsichtigte, wurde niemals realisiert. ${ }^{7}$ Zwei weitere Nachtstücke ohne nachweisliche Verbindung zu Verlaine finden sich in Debussys späterer Klaviermusik: Das mittlere Stück der zweiten Images-Trilogie für Klavier (1907) ist betitelt mit Et la lune descend sur le temple qui fut; und im zweiten Buch der Préludes pour piano (1912/13) verwendet Debussy für das siebte Stück, eine ebenfalls äußerst fragile und zarte Komposition, den nachgestellten Titel La terrasse des audiences du clair de lune.

\section{Clair de lune}

Votre âme est un paysage choisi

Que vont charmants masques et bergamasques,

Jouant du luth et dansant et quasi

Tristes sous leurs déguisements fantasques.

3 Manche Autoren äußern sich skeptisch zum atmosphärischen Einfluss der Watteauschen Malerei auf Verlaines Dichtung. Vgl. Wright 1967, 627 f., sowie Walker 1972, $1107 \mathrm{f}$.

4 Zwischen der ersten und zweiten Vertonung liegt diejenige Gabriel Faurés von 1887 (op. 46 Nr. 2).

5 Wright 1972, 633. Die vierhändige Petite Suite (1889) verwendet zudem mit En bateau und Cortège zwei Gedichttitel von Verlaine als Satzüberschriften (vgl. Wenk 1976, 23).

6 Ebd. Wenk stellt eine konkrete Verbindung her zu zwei Figuren der commedia dell' arte, Brighella und Arlecchino, die aus Bergamo stammen. Gabriel Fauré bezog sich in seiner aus früheren Werken collagierten Theatersuite Masques et bergamasques op. 112 nochmals explizit auf die Sphäre der Fêtes galantes.

7 Wright 1967, 634. Debussy hatte bereits ein eigenes Libretto namens Masques et bergamasques verfasst. 
Tout en chantant sur le mode mineur L'amour vainqueur et la vie opportune, Ils n'ont pas l'air de croire à leur bonheur Et leur chanson se mêle au clair de lune,

Au calme clair de lune triste et beau, Qui fait rêver les oiseaux dans les arbres Et sangloter d'extase les jets d'eau, Les grands jets d'eau sveltes parmi les marbres.

\section{Mondlicht}

Von eigner Art ist deine Seele, wie ein Gefild', in dem Maskierte schreiten, die, tanzend und zur Lautenmelodie, gleichsam ihr Leid mit Masken kleiden.

In Moll erklingen ihre Lieder von Amors Sieg und Glücklichsein. Doch ihre Mienen spiegeln Glück nicht wider und ihr Gesang geht in das Mondlicht ein,

ins stille Mondlicht, schön und voll der Trauer, das Vögel träumen lässt, hoch in den Bäumen, und die Fontänen schluchzen lässt vor Schauer, Fontänen, schlank und hoch, in Marmorräumen.

(Übertragung aus dem Französischen von Bertram Kottmann)

\section{Die Textdichter des jungen Debussy}

Debussy tritt zuerst als Liedkomponist an die Öffentlichkeit. Der junge Komponist vertont zunächst vorrangig französische Romantiker, darunter Alfred de Musset und Théodore de Banville. Die meisten der zahlreichen mélodies der 1880er Jahre werden für die Sopranstimme von Debussys Gönnerin Marie Vasnier komponiert, der Gattin eines Pariser Architekten, die den jungen Musiker fördert und ihn mit zeitgenössischen französischen Schriftstellern bekannt macht. Nach seinen Russlandaufenthalten in den Sommern 1881 und 1882 auf Einladung Nadeschda Filaretowna von Mecks, durch die er die Musik des >Mächtigen Häufleins` kennen lernt, wendet sich Debussy erstmals der Lyrik Paul Verlaines zu und vertont für Madame Vasnier einige Gedichte aus den Fêtes galantes. Bis Ende 1882 entstehen die frühen Versionen von Fantoches (seine erste Verlaine-Vertonung), En sourdine und Clair de lune', außerdem Mandoline und Pantomime sowie

8 Die Deutsche Gedichtebibliothek, http://www.gedichte.xbib.de/uebersetzung_schreiben_Verlaine_Mondschein_1.htm

9 Es existieren abweichende Meinungen darüber, wie viele Verlaine-Gedichte Debussy mehrmals vertont hat. Roger Nichols nennt nur En sourdine und Clair de lune; Iris Helene Hilbk spricht hingegen von acht (!) Gedichten. Vgl. Nichols 1967, 229, sowie Hilbk 1996, 57. 
eine Vertonung von Banvilles Gedicht Fête galante, das den Verlaine-Liedern an die Seite gestellt werden kann. Die sogenannte Vasnier-Gruppe ${ }^{10}$ umfasst Pantomime, Clair de lune, Pierrot (1881, nach Banville) und Apparition (1884, nach Mallarmé) und erscheint zu Debussys Lebzeiten niemals im Druck; die vier Jugendlieder werden erst 1926 in der Zeitschrift Revue musicale publiziert und firmieren in einer späteren Ausgabe bei Jobert (1969) unter dem Titel Quatre chants de jeunesse.

Insgesamt basiert ein gutes Drittel von Debussys mélodies, nämlich neunzehn Lieder, auf Gedichten Verlaines. Ab 1883 wendet sich Debussy zunächst den Dichtern Stéphane Mallarmé, Paul Bourget und Édouard Guinand zu; Verlaine vertont er erst wieder 1885 in den Ariettes oubliées. Nach seinem Rompreis-Aufenthalt in der Villa Medici folgt 1887 der von intensiver Wagner-Rezeption geprägte Zyklus Cinq poèmes de Baudelaire. In der Folge komponiert Debussy vorrangig Instrumentalmusik, bis 1891 wieder eine ausgiebige Verlaine-Phase einsetzt. Das erste Heft der Fêtes galantes entsteht (mit überarbeiteten Versionen der bereits 1882 vertonten Fantoches und En sourdine sowie einer vollständigen Neukomposition von Clair de lune) - außerdem veröffentlicht Debussy Trois mélodies nach Verlaines Sagesse-Zyklus. Das zweite Triptychon der Fêtes galantes (welches die Gedichte Les ingénus, Le faune und Colloque sentimental umfasst) wird erst 1904 publiziert und markiert den Endpunkt von Debussys Auseinandersetzung mit der Lyrik Verlaines. ${ }^{11}$

\section{Analytische Betrachtung}

Ein Vergleich der beiden Versionen von Clair de lune (1882 und 1891) zeigt einen signifikanten Wandel der Tonsprache Debussys. Während die frühe Vertonung als ein Beispiel formal konventioneller, über den harmonischen Rahmen der mélodies von Fauré und Henri Duparc kaum hinausweisender Liedkomposition gelten kann, demonstriert das spätere Lied einen reifen Personalstil und einen die Textvorlage innovativ umsetzenden Zugriff. ${ }^{2}$ Man muss nicht, wie Roger Nichols oder Barbara Meister, die frühere Version als unreif oder als von Mängeln behaftet ansehen ${ }^{13}$, um den kompositionstechnischen Fortschritt während der neun Jahre, die beide Werke trennen, zu bemerken. Ohne Frage erscheint die spätere Fassung aber für die Analyse ergiebiger, weshalb sie hier im Mittelpunkt der Aufmerksamkeit steht.

10 So werden die vier Lieder referenziert bei Thompson 1937, 286. Zur Zusammensetzung des VasnierAlbums siehe auch Hardeck 1967, $12 \mathrm{f}$.

11 Die Wesensverwandtschaft zwischen beiden Künstlern wird von Theo Hirsbrunner $(1981,164)$ betont, der aus Verlaines Gedicht Art poétique zitiert und schließt, dass diesem für Vertonungen seiner Verse eine »in die Dichtung eindringende« und »fein abgetönte Musik» am Herzen gelegen habe.

12 Im Gegensatz hierzu steht die Behauptung Erwin Hardecks, der heranwachsende Komponist habe den symbolischen Gehalt der Fêtes galantes noch nicht vollständig erschließen können und deshalb erneut auf die drei Gedichte zurückgegriffen (1967, 162).

13 Dieses Urteil äußert sich in Zuschreibungen wie »the harmony [...] is quite unremarkable (Nichols $1967,233)$ oder gar »the setting [...] destroys the music of the poem « (ebd., 235). Die frühe Fassung wird als »immature effort " bezeichnet (Meister 1998, 340) und im Vergleich mit den anderen Liedern der Vasnier-Gruppe als schwächer dargestellt (ebd., 283). 
Debussy bedient sich in seiner mittleren Schaffensphase des zwölfstufig temperierten Tonsystems in einer Weise, welche die Hochalteration einer diatonischen Stufe synonym zur Tiefalteration ihres oberen Nachbartons verwendet. Gelegentlich werden Kreuz- und B-Tonarten vermischt, ohne dass dies eine harmonische (oder semantische) Bedeutung implizieren würde. Vielmehr wird lediglich die Notation vereinfacht (etwa T. 8: im Klavierpart wird $\mathrm{B}^{7}$ statt $\mathrm{Ais}^{7}$ notiert, in der Singstimme jedoch ais und eis; T. 12: über Dis ${ }^{7}$ erklingen in der Singstimme $f$ und $g$, die fallende verminderte Quarte $g$-dis ist in Wahrheit eine große Terz fisis-dis). Dadurch erscheinen einzelne Töne und Akkorde im Notenbild entlegener, als sie es tatsächlich sind. Mitunter werden auch Leittöne nicht korrekt notiert, um eine doppelte Vorzeichnung zu vermeiden (etwa in der Singstimme in Takt 20-22: $d$-dis-d-dis). In der folgenden Analyse setze ich durchgehend orthographische und harmonische Enharmonik voraus, um systematische Schlüsse aus Tonfolgen und Zusammenklängen ziehen zu können, und verweise mit Gleichheitszeichen auf die enharmonische Identität von Einzeltönen oder Akkorden.

\section{Aspekte der Melodik}

Das Lied besitzt eine pentatonische Klaviereinleitung, welche nur die Töne dis, fis, gis, ais, cis und damit ausschließlich schwarze Tasten verwendet; die Unterstimme umkreist dis und ais und sorgt zunächst für eine stabile Tonalitätswahrnehmung. Der suggerierte Grundton dis erweist sich jedoch nicht als dauerhaft: Mit dem Einsatz der Singstimme (T. 5) wird überraschend gis zum Bezugspunkt der melodischen Entwicklung, ohne dass diese Wendung aus dem leittonlosen Material der pentatonischen Skala hätte antizipiert werden können. ${ }^{14}$ Das Ende des Liedes verweist auf dessen Beginn: Ohne wieder auf die Pentatonik der Einleitung zurück zu kommen, setzt in Takt 27, ausgehend vom melodischen Hochpunkt fis ${ }^{2}$, eine diatonisch absteigende Linie an, die alle Tonleiterstufen von dis-Moll berührt und schließlich eine Dezime tiefer in den Schlusston dis ${ }^{1}$ mündet (T. 30). Hier stellt der Klavierpart, ähnlich ansatzlos wie zu Beginn, die Grundtonalität gis-Moll wieder her.

Die melodische Bewegung ist durchweg engschrittig. Die Singstimme wird über weite Strecken diatonisch geführt; trotz einzelner Tendenzen zur Chromatisierung (T. 7 und 8: eis-e; T. 26-27: f-fis) besitzen diatonische Skalenausschnitte und Dreiklangsbrechungen vorrangige melodische Relevanz. Zu Beginn der ersten Strophe (»Votre âme est un paysage choisi«, T. 5 und 6) umkreist die Singstimme gemeinsam mit der >colla parte` geführten Klavier-Unterstimme den Ton e (Beispiel 1a). Das hier ausgebreitete Tetrachord dis-e-fis-gis ließe sich, an die Einleitung anknüpfend, in dis-phrygisch verorten, wird aber durch den Klavierbass unmissverständlich in gis-Moll fundiert. Die Töne $c$ und $g$, halbtönig benachbart zu $h$ und fis (T. 11 und 12), prägen eine Nebentonalität aus, die durch enharmonische Gleichsetzung von $g$ und fisis (Takt 12) wieder ins Lot gerückt wird. Eine Linienchromatik findet sich lediglich im Klavierbass von Takt 7; in der ausgeterzten

14 Nichols $(1967,231)$ weist darauf hin, dass der Übergang Takt 4-5 einer Kadenzwirkung entbehre "partly because the leading-note is flattened". Dieser Befund ist irreführend, da pentatonisches Material per se eines Leittons entbehrt. 
Tonfolge $c i s$ - $d$-dis- $d$-cis folgen die Alterationen nicht der melodischen Richtung, sondern einer autonomen Logik benachbarter Klangtypen.
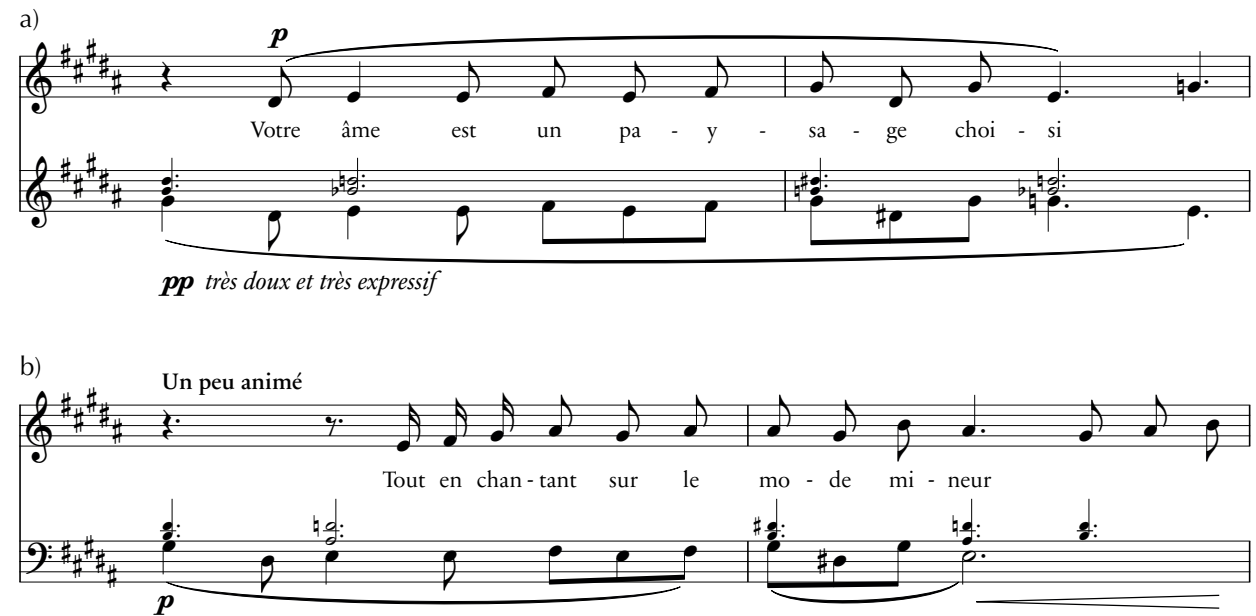

Beispiel 1: Claude Debussy, Clair de lune (1891), a) T. 5-6, b) T. 13-14 (reduzierter Klaviersatz)

Die zweite Strophe (»Tout en chantant« ab T. 13) zitiert im Klavier den Melodiebeginn der ersten Strophe (Beispiel 1b), während die kontrapunktierende Singstimme den Ton gis umkreist und für vier Takte in der gis-Moll-Diatonik verweilt. Eine konsistente motivische Analyse erscheint allerdings kaum möglich. Zwar ist der Einschnitt zwischen erstem und zweitem Vers durch die melodische Reprise zweifelsfrei identifizierbar, in der Folge werden Syntax und Gesamtform der Komposition aber mehr durch harmonische Gegebenheiten als durch Motivstrukturen konstituiert. ${ }^{15}$ Ein erster melodischer Höhepunkt fis $^{2}$ wird bei der Textzeile »lls n'ont pas l'air« (T. 17) erreicht; gebrochene Akkorde der Singstimme in h-Moll und g-Moll (T. 19-20) stehen quer zur Grundtonart, ergänzen aber das akkordische Fundament des Klaviersatzes um charakteristische Optionstöne. Auch die dritte Strophe, im zarten Pianissimo ansetzend und den längsten Spannungsbogen des gesamten Liedes darstellend, gewinnt ihre Melodik vorrangig aus Akkordbrechungen (T. 21-22: gis-Moll bzw. gis ${ }^{7}$, T. 24-25: Fis $^{7}$ ). Nachdem Debussy auf direkte Wiederholungen weitestgehend verzichtet, fällt die melodische und harmonische Übereinstimmung der Takte 23 bis 25 besonders ins Gewicht.

15 Aus diesem Grund greift Erwin Hardecks Analyse des Liedes, welche sich auf drei aus dem Text

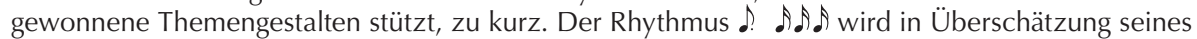
semantischen Gehalts als "gespenstisches Menuettmotiv« bezeichnet, während die beiden anderen Motive kaum nachzuverfolgen sind (1967, $160 \mathrm{ff}$.$) .$ 


\section{Aspekte der Metrik, Syntax und Form}

Das Gedicht besteht aus drei Strophen mit jeweils vier vierhebigen Zeilen, die in regelmäßigen Kreuzreimen angeordnet sind. Die 32 Takte umfassende Vertonung orientiert sich in ihrem Gesamtverlauf eng an der Textvorlage - es sind drei nahezu gleich lange Strophen identifizierbar (beginnend in T. 5, T. 13 und T. 21), in denen jede Zeile durchschnittlich zwei Takte einnimmt. Die metrische Organisation ist jedoch unvorhersehbar: Im stetigen 9/8-Takt fallen betonte Silben immer auf unterschiedliche Zählzeiten, und ein einheitliches musikalisches Metrum wird vermieden zugunsten einer prosaischen, stets syllabischen Rhythmik der Singstimme, welche zwischen Zusammenfassungen mehrerer kurzer Silben und stark gedehnten Noten frei alterniert. Häufig beginnen Textzeilen nicht auf der ersten Zählzeit; auf diese Weise werden Taktschwerpunkte überspielt, und Taktstriche werden weniger durch rhythmische Einheitlichkeit denn durch Harmoniewechsel kenntlich gemacht. Dass im Ganzen dennoch zweitaktige, das Reimschema des Gedichts verdeutlichende Gruppen vorherrschen, kann als eine besondere, für Debussys reife Vokalmusik charakteristische Form der Vereinigung von metrischer Freiheit mit syntaktischer Regelmäßigkeit angesehen werden. Das Enjambement zwischen zweiter und dritter Strophe, welches mit der Wiederholung der titelgebenden Worte »clair de lune» korrespondiert, findet eine direkte Reflexion in der Musik: Die Takte 20 und 21 werden weder melodisch noch syntaktisch getrennt, die Singstimme rückt ohne Atempause von $d$ nach dis, und der Strophenbeginn wird lediglich durch die Rückkehr zur Ausgangstonalität gis-Moll angezeigt.

Die melodischen Phrasen der Singstimme enden bevorzugt auf langen, mitunter den Takt ausfüllenden Notenwerten. Am deutlichsten ist dies in der zweiten Hälfte der zweiten Strophe (T. 17-18 und 19-20): Hier fällt der Zeilenbeginn erstmals auf die Takt-Eins, und beide Zeilen entsprechen sich rhythmisch vollständig, als solle die Wirkung der extravaganten Harmonik (von der noch zu sprechen sein wird) durch eine unerwartet regelmäßige, im Klaviersatz gar repetitive Rhythmisierung abgeschwächt werden. ${ }^{16}$ Durch die Symmetrie dieser beiden Taktpaare wird die achttaktige Kongruenz der beiden ersten Strophen vollendet. Die sich anschließende dritte Strophe hingegen zeigt sich auf zehn Takte erweitert; dies geschieht nicht allein durch eine extreme Dehnung der Schlussphrase $^{17}$, sondern bereits innerhalb der ersten Zeile »Au calme clair de lune» (T. 21), die aus der steten Zweitaktigkeit ausbricht und mit den Worten striste et beau « in einen frei ausgestalteten, nicht mehr weiter unterteilbaren Melodiebogen überleitet.

Eine rhythmische Figur, die aus der Einleitung stammt (T. 1-2), kehrt im Klaviersatz

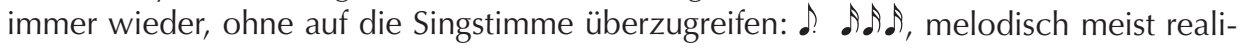
siert durch eine Auf- und Abwärtsbewegung in Sekunden. Innerhalb des Liedes taucht dieser Leitrhythmus in der Mittelstimme in Takt 9-10 wieder auf und ergänzt eine in Takt 8 exponierte Tremolofigur in Doppelgriffen der rechten Hand, die ebenfalls häufig

16 Dies wird betont von Roger Nichols, der eine auffällige Korrespondenz der beiden Zeilen im Hinblick auf Rhythmik und Textaussage beobachtet. Ebenso verhält es sich in den Takten 27-28, hier bei gleichbleibender Harmonik (vgl. 2003, 89).

17 Ebd. Der Dehnung des Schlusswortes »marbres« wird eine maßgebliche emotionale Wirkung zugeschrieben. 
wiederkehrt. ${ }^{18}$ Der Leitrhythmus dominiert später die Takte 16 und 19 bis 20 sowie weite Teile der dritten Strophe, ehe er sich in die kontinuierlichen Sechzehntelfigurationen der Tremolofigur auflöst (ab T. 26), welche bis zum Schluss des Liedes nicht mehr abbrechen. ${ }^{19}$

\section{Aspekte der Tonalität und Harmonik}

Das Lied besitzt, ungeachtet seiner in dis fundierten, moll-pentatonischen Einleitung und des wieder auf dis-Moll rekurrierenden Schlussteils, eine über weite Strecken unangefochtene Tonalität: Mit dem ersten Einsatz der Singstimme kristallisiert sich gis-Moll als Grundtonart heraus. ${ }^{20}$ Diese wird einerseits durch ihre diatonischen Nebenstufen flankiert und operiert mit tradierten Kadenzwendungen. Andererseits führt Debussy an einigen Stellen alterierte Wechselakkorde ein; dies sind vorrangig aus einer Ganztonskala über gis gewonnene Vierklänge, welche zu vorübergehenden Änderungen des Klangzentrums (insbesondere mit den Grundtönen $d, c$ und $b$ ) führen. Für die resultierenden Modulationen etablieren sich exterritoriale, funktionsharmonisch nicht legitimierbare Farbwechsel und Klangfolgen. Mit dieser Charakterisierung nimmt das Lied eine Mittelposition ein zwischen der weitgehend dur-moll-tonal gefestigten Dreiklangsharmonik des frühen Debussy und der experimentellen, verschiedenste Mittel der Polymodalität, Akkordmixtur und chromatischen Rückung gebrauchenden Spätphase. In Clair de lune wird Tonalität im Sinne einer übergeordneten oder mindestens passagenweise definierten Grundtonbezogenheit niemals in Frage gestellt.

Es existiert eine formbildende, durch die Strophenstruktur des Liedes bedingte Logik der harmonischen Progression. Jede Strophe beginnt tonikal im Piano, in der dritten Strophe (T. 21) mit der Septime eines gis-Mollseptakkords im Bass. ${ }^{21}$ Im Strophenverlauf finden jeweils Ausweichungen statt, gekoppelt mit einem Ansteigen der Tonhöhe oder einem Anschwellen der Lautstärke:

18 Hilbk (1996, 137 f.) definiert in einer kursorischen, die Harmonik vernachlässigenden Besprechung des Liedes ebenfalls zwei verschiedene rhythmische Muster und betont die Abstammung des Leitrhythmus aus der Einleitung.

19 Nichols $(1967,230)$ beobachtet eine Hervorhebung des Wortes »sangloter « durch die in Takt 29 vorangehende, für die Dauer dreier Achtel innehaltende Sechzehntelbewegung.

20 Verschiedentlich wurde die Textzeile »tout en chantant sur le mode mineur entgegen der vielschichtigen Semantik von »mineur in Zusammenhang mit dem Tongeschlecht der Komposition gebracht. So nennt Wenk (1976, 25) drei mollare Modi (dis-Moll-pentatonisch, gis-äolisch zu Beginn der ersten Strophe und gis-dorisch am Schluss des Liedes) als Belege für den musikalisch-semantischen Einfluss der Textzeile »sur le mode mineur». Hilbk (1996, 136) bezeichnet die frühe Fassung von Clair de lune unrichtig als Moll-Komposition. Auch das Klavierstück Clair de lune aus der Suite bergamasque steht nicht in Moll (Des-Dur).

21 Dieser zwischen Dur und Moll changierende Vierklang kann auch als H-Dur-Quartsextakkord mit großer Sexte gedeutet werden. Um die tonale Kongruenz der Strophenanfänge zu bestätigen, gebe ich dem Grundton gis den Vorzug, obgleich der Mollseptakkord durch die im Bass liegende Septime destabilisiert erscheint. 
- Erste Strophe: Bei »bergamasques« (T. 8) wird ein doppeldominantischer Terzquartakkord $\mathrm{B}^{7}$ erreicht. ${ }^{22}$ Wenig später folgt bei "tristes« (T. 11) ein vagierender, auf $d$ basierender Ganztonakkord.

- Zweite Strophe: In Takt 17 bis 18 etabliert sich ein stabiles D-Dur (verkörpert durch die Akkorde D6 und Dmaj7), das in einen dominantischen Klang $\mathrm{C}^{7 / 9}$ fortgeführt wird.

- Dritte Strophe: Bei »sangloter d'extase« (T. 26) erklingt ein tonikales B-Dur (mit Nebenstufe c-Moll).

Diese Ausweichstationen liegen sämtlich auf einer Ganztonachse über gis, und zwar in möglichst großer tonaler Entfernung. In einer Molltonart können grundstellige Akkorde auf der hochalterierten III. oder IV. Stufe funktional nicht zugeordnet werden; B-Dur = Ais-Dur könnte zwar als Doppeldominante eingesetzt werden, prägt aber in Takt 26 ein eigenes tonikales Subsystem aus und wirkt dadurch exotisch. Hingegen sind Akkorde mit der Basis e und fis, die ebenfalls innerhalb der Skala liegen (etwa in T. 23: $\mathrm{Fis}^{7 / 9}$ bzw. Fis ${ }^{13}$ ), ohne Umstände als leitereigene Stufen in gis-Moll oder H-Dur deutbar. Betrachtet man diejenigen Akkorde, deren Grundtöne nicht auf der genannten, sondern der komplementären Ganztonachse über dis liegen, so können diese ebenfalls einem Kadenzumfeld von gis-Moll zugeordnet werden (etwa in T. 10: $\mathrm{cis}^{7 / 9}$, T. 12: Dis-Dur, T. 29: H-Dur), während die potentiell entlegeneren Stufen aus dieser Ganztonachse, nämlich $a$, eis $=f$ und fisis $=g$ im ganzen Lied ausgespart werden (mit einer Ausnahme in T. 23-24, wo F ${ }^{7}=$ Eis $^{7}$ als Wechselklang mit $\mathrm{Fis}^{7 / 9}$ alterniert, allerdings keine tonikale Stufe ausprägt). Debussy bedient sich an den genannten Stellen also ausschließlich grundstelliger Dur-Dreiklänge aus der Ganztonachse über gis (namentlich $A i s=B, C$ und $D$ ), weil diese geeignet sind, die Dur-Moll-Tonalität zu unterwandern.

Zu Beginn der ersten Strophe lösen sich das tonikale gis-Moll (T. 5.1 und 6.1) und zwei in ihrer Substanz sehr ähnliche Vierklänge über e gegenseitig ab. Der erste Wechselklang (T. 5.2-3) besteht aus Tönen der erwähnten Ganztonachse, verzichtet aber auf gis und his $=c$; der resultierende Akkord e-fis- $b=$ ais- $d=$ cisis besitzt in seinen Außenstimmen zwei Leittöne ( $d$ =cisis und e führen in Gegenbewegung zu dis zurück) und ließe sich in Gestalt eines übermäßigen Sekundakkords als Zwischendominante (Fis ${ }^{7}$ mit hochalterierter Quinte) darstellen. ${ }^{23}$ Diesem Klang entspricht das spitch class set $[0,2,4,8]$ mit der Ordnungszahl 4-24. ${ }^{24}$ Der zweite Wechselklang (T. 6, 2-3) ersetzt fis durch $g$, unterscheidet sich vom ersten also nur in einem einzigen Ton, so dass ein (ebenfalls doppelt leittöniger) halbverminderter Septakkord über e entsteht e- $g-b=a i s-d=c i s i s$. Hier entsteht das spc-set، $[0,2,5,8]$ mit der Ordnungszahl 4-27. Die kontrapunktische Strebe-

22 Barbara Meister $(1998,340)$ missversteht den letzten Klang in Takt 7 als »diminished chord based on $A \#$ «, um eine Großterz zu einem halbverminderten Septakkord erweitert, statt diesen Akkord als bloßen Wechselklang zum vorangegangenen gis-Moll zu identifizieren. Den Eintritt des doppeldominantischen $\mathrm{B}^{7}$ bezeichnet sie als »effect [...] of being lifted harmonically«, ohne den damit verbundenen Anstieg um zwei Quinten zu benennen.

23 In Takt 13 wird der gleiche Akkord ohne erkennbaren Grund mit ais statt $b$ notiert (vgl. Beispiel 1).

24 Die im Text erwähnten spitch class sets` entsprechen jeweils der sprime form` des genannten Akkords nach Forte 1973. 
wirkung der beiden auf dis gerichteten Außenstimmen ist jeweils so unverkennbar, dass beide Akkorde als entlegene Varianten einer phrygischen Wendung mit übermäßigem Quintsext- oder Terzquartakkord gehört werden können (siehe Beispiel 2). Zu Beginn der zweiten Strophe kehrt der erste Wechselklang über einem Orgelpunkt gis wieder und verwandelt sich schließlich durch den Schritt ais- $h$ tatsächlich in einen übermäßigen Quintsextakkord (T. 14.3).

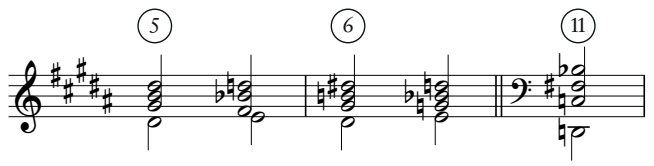

Beispiel 2: Claude Debussy, Clair de lune (1891), T. 5, 6 und 11, Akkord-Extrakt

Ein verwandter, ebenfalls dem spc-set $[0,2,4,8]$ entsprechender Akkord erklingt zu dem Wort »tristes« (T. 11.1-2): Nun werden vier Töne aus der Ganztonachse über gis als alterierter Tredezimakkord über $d$ dargestellt $d$-fis- $b=$ ais-c. ${ }^{25}$ Nachdem in Takt 10 die Nebenstufe $\mathrm{H}$-Dur kadenziell bestätigt wurde, ist dieser Klang ein durchaus überraschendes Ereignis. Ebenso abrupt geht er in den übermäßigen Dreiklang es $=$ dis- $g=$ fisis- $h$ über (T. 11.3), welcher durch den folgenden, vollständig von Dis-Dur eingenommenen Takt 12 eindeutig als alterierte Dominante erkennbar wird. Der erwähnte $D^{13}$ (mit hochalterierter Quinte $b=$ ais) könnte optional auch aus der akustischen Skala über $c$ abgeleitet werden; er steht für sich, da seine Fortführung durch den Bassverlauf $d$-g kaum als Quintfall oder als ein anderer Fundamentschritt gehört werden kann.

Verknüpfungen in Form von Quintschritten spielen insgesamt eine eher untergeordnete Rolle (eine klare V-I-Verbindung erscheint etwa beim Übergang T. 12-13), scheinen aber, wenn sie vorkommen, die Dur-Moll-Tonalität gleichsam als Kontrast zur chromatischen oder ganztönigen Alterationsharmonik konstituieren zu wollen. Dies geschieht bei »ouant du luth et dansant" (T. 9-10), wo über die Fundamente dis-gis-cis-[e]-fis nach $\mathrm{H}$-Dur kadenziert wird. ${ }^{26}$ Eine gewisse Relevanz besitzen leittonlos-modale Verbindungen von grundstelligen Akkorden im Sekund- oder Terzabstand, so etwa I-ii (T. 26: B-Dur alterniert mit seiner Nebenstufe c-Moll). Die Grundtöne einer absteigenden Kette leitereigener Dreiklänge dis-cis-h-gis, wie sie sich in Takt 28 bis 30 entfaltet, scheinen aus der pentatonischen Sphäre der Einleitung abgeleitet zu sein. ${ }^{27}$

Die harmonisch avancierteste Passage findet sich am Ende der zweiten Strophe (T. 17-20; siehe Beispiel 3). Zu Beginn von Takt 17 wird kurz ein tonikales Zentrum auf dem Orgelpunkt $d$ installiert, das aber noch im Verlauf desselben durch Hinzufügung von Sexten und Septimen destabilisiert wird. Die Klangfolgen der sich anschließenden Taktübergänge (T. 18-19 und 20-21) sind höchst unerwartet; als Trugschlüsse wären sie unzureichend beschrieben, da sie weder auf traditionelle Leittonverbindungen noch

25 Wenk $(1976,29)$ stellt eine semantische Assoziation des Ganztonakkords mit dem Wort »tristes « her: »the whole-tone harmonization [...] conveys a desolation which goes beyond the quasi of the text«.

26 Nichols 1967, 233.

27 Bei Thompson $(1937,294)$ heißt es: «the accompaniment is of modal coloring, with unexpected transitions «. Diese allgemein gehaltene Aussage scheint auf derartige Akkordverbindungen abzuzielen. 
auf Beziehungen von Fundamentschritten rekurrieren, sondern einzig durch frei chromatische Stimmführung auseinander hervor gehen. Die elliptische Verknüpfung der jeweils zwei Takte anhaltenden Orgelpunkte auf $d, c$ und fis sträubt sich gegen jegliche funktionsharmonische Deutung - sie werden als eigenständige Farbwerte gegeneinander gestellt. Am Übergang der zweiten zur dritten Strophe wendet sich der Tonsatz unvermittelt nach gis-Moll bzw. gis ${ }^{7}$ zurück, wodurch die Worte »clair de lune», die zweimal erscheinen (T. 20 und 21-22), jeweils in einer anderen harmonischen Sphäre verortet werden. Trugschlüssige Wendungen im traditionellen Sinn erscheinen im Lied dennoch an anderen prägnanten Stellen: Takt 16 zielt als ii-V-Kadenz auf H-Dur, das nicht erscheint; an dessen Stelle erklingt D-Dur. Die Takte 23 bis 25 bilden eine Dominantfläche über dem Orgelpunkt fis, die ebenfalls H-Dur erwarten lässt, aber überraschend in B-Dur mündet.

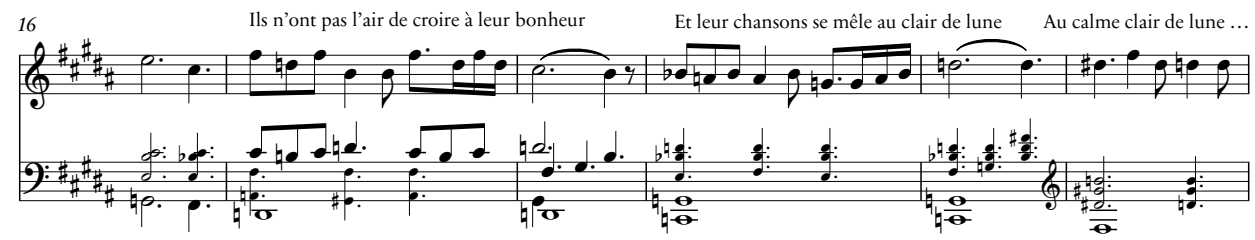

Beispiel 3: Claude Debussy, Clair de lune (1891), T. 16-21: Übergang von der zweiten zur dritten Strophe (reduzierter Klaviersatz)

Der Schlussteil kann als eine Versöhnung der tonalen Eskapaden der Mittelstrophe sowohl mit der Grundtonart gis-Moll, als auch mit der Pentatonik der Einleitung angesehen werden. Die Takte 27 und 28 sind klar in dis-Moll fundiert; bei "parmi les marbres" (T. 29) scheinen die absteigenden Akkorde Cis-Dur und H-Dur ein fallendes phrygisches Tetrachord anzudeuten und auf einen Akkord über ais zu zielen, der durch den erneuten Eintritt von gis-Moll in Takt 30 allerdings umgangen wird (bereits in T. 26 war Ais-Dur = B-Dur erklungen). Wieder wird die Tonika leittonlos, mit Quintparallelen in den Außenstimmen ( $h$-fis und gis-dis), erreicht; die Klangfolge dis-Cis-H-gis erweitert die Grundtonart zur Moll-Pentatonik. Indessen wird die gedehnte Schlussphrase der dritten Strophe, mit einem Tremolo in gis-Moll verebbend, durch die große Sexte eis dorisch eingefärbt. ${ }^{28}$ Auf die tonale und atmosphärische Verwandtschaft, welche den Schluss des Liedes mit einem anderen, einige Jahre zuvor entstandenen Verlaine-Lied Debussys verbindet (II pleure dans mon cœur aus den Ariettes oubliées von 1888), ist bereits an anderer Stelle hingewiesen worden. ${ }^{29}$

28 Wright $(1967,630)$ versucht den Ton eis, der sowohl im Kontext von dis-Moll (T. 27) als auch innerhalb der in gis-Moll fundierten Takte 29 bis 31 begegnet, auf die Grundtonart zu beziehen und gibt die Tonalität der Schlusstakte mit gis-hypodorisch an (vgl. auch Wenk 1976, 25).

29 Vgl. Nichols 1967, 233. 


\section{Semantik und Symbolismus}

In dem besprochenen Lied lassen sich über das titelgebende »clair de lune« hinaus noch einige weitere Wort-Ton-Beziehungen ausmachen. Das Wort »déguisements« (T. 11-12) trifft sich mit einer enharmonisch ıverkleideten`, nach gis-Moll zurückführenden Dominante; die Klangtäuschungen bei »ils n'ont pas l'air de croire à leur bonheur« (T. 17-18) sind wohl nicht zufällig mit einer Textpassage verbunden, die einen metaphorischen Kontrast zwischen Gefühlen und äußerem Erscheinungsbild der poetischen Figuren herstellt. ${ }^{30}$ Die affektgeladene Zeile »sur le mode mineur« könnte ein (wenn auch spekulatives) Argument abgeben, warum Debussy den Leitton cisis nicht als Hochalteration der vierten Skalenstufe, sondern harmonisch inkorrekt als $d$ notiert - dieses Phänomen vermag sowohl als Folge eines einzeltonalen smode mineurı, also einer Tiefalteration, als auch vor dem Bedeutungshintergrund von smineur als ızweitrangig bzw. `defizitär schlüssig erscheinen. Auch das zweifellos leittönig aufzufassende Alternieren der Singstimme zwischen $d$ und dis zu den Worten »clair de lune« (T. 20-22) könnte sich dieser semantischen Konnotation verdanken: Die Klausel-Penultima $d=$ cisis fungiert als eine textbedingte Eintrübung von dis. ${ }^{31}$ Schließlich findet auch das beschließende »sangloter d'extase les jets d'eau", das leidenschaftliche Schluchzen der Wasserfontänen, in der klagenden, über eine Dezime absteigenden Gesangslinie der Takte 27 bis 30 seine musikalische Entsprechung.

Der Umstand, dass Debussy sich in seinem kompositorischen Schaffen über zwei Jahrzehnte immer wieder auf wenige Zeilen eines einzigen Gedichts bezog, ist faszinierend. Diese Komposition darf als seine gelungenste Umsetzung der literarischen Vorlage gelten - in der Gesamtwahrnehmung des Liedes vermittelt sich, in vollkommener Entsprechung zu der Textzeile »triste et beau«, eine allgemeine Atmosphäre trauriger Schönheit. Diese lässt das musikalische Mondbild in einer faszinierenden affektiven Geschlossenheit wirken, deren sanftem Zauber selbst die beschriebenen tonalen Ausbrüche nichts anhaben können.

\section{Literatur}

Forte, Allen (1973), The Structure of Tonal Music, New Haven: Yale University Press.

Hardeck, Erwin (1967), Untersuchungen zu den Klavierliedern Claude Debussys, Regensburg: Gustav Bosse.

Hirsbrunner, Theo (1981), Debussy und seine Zeit, Laaber: Laaber.

Hilbk, Iris Helene (1996), Studien zum Verhältnis von Sprache und Musik bei Debussy. Eine Untersuchung ausgewählter Werke vor dem Hintergrund seiner Briefe, Feuilletons und Musikkritiken, Frankfurt a.M. u.a.: Peter Lang.

30 Vgl. Walker 1972, 1009.

31 In diesem Zusammenhang sind die Ausführungen von Arthur Wenk aufschlussreich, der sich detailliert zu Verlaines Zuordnung des Adjektivs mineur im Kontrast zu der Textpassage »la vie opportune« äußert (1976, 25 ff.). 
Lesure, François (1977), Catalogue de l'œuvre de Claude Debussy, Genf: Editions Minkoff.

Meister, Barbara (1998), Nineteenth-century French Song: Fauré, Chausson, Duparc, and Debussy, Bloomington: Indiana University Press.

Nichols, Roger (1967), »Debussy's Two Settings of Clair de lune«, Music and Letters 48/3, 229-235.

Nichols, Roger (2003), »The Prosaic Debussy«, in: The Cambridge Companion to Debussy, hg. von Simon Trezise, Cambridge: University Press, 84-100.

Schmidt, Christian Martin (1991), »Mahler, Reger, Schönberg: Nachtstücke», in: Nationaler Stil und europäische Dimension in der Musik der Jahrhundertwende, hg. von Helga de la Motte-Haber, Darmstadt: Wissenschaftliche Buchgesellschaft, 79-85.

Seidel, Elmar (1969), Artikel »Nocturne«, in: Riemann Musiklexikon, Sachteil, Mainz: Schott, $635 \mathrm{f}$.

Thompson, Oscar (1937), Debussy: Man and Artist, New York: Dodd, Mead \& Company, Reprint New York: Dover Publications 1967.

Walker, Hallam (1972), „Visual and Spatial Imagery in Verlaine's Fêtes galantes«, PMLA 87/5, 1007-1015.

Wenk, Arthur B. (1976), Claude Debussy and the Poets, Berkeley: University of California Press.

Wright, Alfred J. (1967), „Verlaine and Debussy: Fêtes galantes«, The French Review 40/5, 627-635. 